\title{
Inverse determinations of thickness and elastic properties of a bonding layer using laser-generated surface waves
}

\author{
T.-T. Wu *, Y.-H. Liu \\ Institute of Applied Mechanics, National Taiwan University, Taipei, Taiwan
}

Received 23 February 1998

\begin{abstract}
A recent study on the dispersion of laser-generated surface waves in an epoxy-bonded copper-aluminum layered specimen showed that the influence of bonding layer thickness on the surface wave dispersion is clear and could be applied to the NDE of bonding properties. In this paper, an inversion algorithm is introduced to determine the thickness and the elastic properties of bonding layer in a bonded layered medium from the measured velocity dispersion of laser-generated surface waves. Laser ultrasonic experiments were performed on an epoxy-bonded copper-aluminum layered specimen with small bonding thickness. The received dispersive elastic wave signals were processed via the spectral analysis method to obtain the experimental dispersion curve. Inverse analyses of one parameter (thickness), two parameters (longitudinal and transverse wave velocities) and three parameters (density, longitudinal and transverse wave velocities) of the epoxy bonding layer were made. Discussions of the convergence and constraints of the inversion process are included. (C) 1999 Elsevier Science B.V. All rights reserved.
\end{abstract}

Keywords: Inverse problem; Laser ultrasonics; Bonding layer

\section{Introduction}

Due to the non-contact feature and the ability of broadband signal generation, laser ultrasonics has demonstrated its great potential for NDE applications [1]. Laser-generated ultrasonic waves have been applied to investigate the Lamb wave propagation phenomena in thin plates [2-4] and to obtain a scan image of thin graphite-epoxy laminates and silicon wafer [5]. Lasergenerated ultrasonic bulk waves [6] and surface waves $[7,8]$ have also been applied to the determination of elastic constants of anisotropic materials. The anisotropic elastic constants were obtained through the measurements of the group velocities of laser-generated surface waves and the use of an inversion algorithm. The elastic constants for composite overlays have been measured by employing a line focused pulsed laser [9]. Both the phase velocities and the skew angles between the phase and the group velocities of the skimming longitudinal waves on the surface have been measured to fit the elastic constants. Laser ultrasonics has also been applied to the study of Lamb wave propagation in

\footnotetext{
* Corresponding author. Tel: (886) 2-2363-0979; fax: (886) 2-363-9290; e-mail: wutt@ndt.iam.ntu.edu.tw
}

Ref. [10]. The results showed that the lowest Lamb wave modes could be identified clearly with minimal damage to the paper. The laser-generated ultrasonic guided waves have recently been applied to the carbon fiber-reinforced polymer composite laminates [11] and good agreements between the calculated and experimental results were reported.

In a recent paper by $\mathrm{Wu}$ and $\mathrm{Chen} \mathrm{[12],} \mathrm{the} \mathrm{dispersion}$ of laser-generated surface waves in an epoxy bonded copper-aluminum layered specimen was studied. Their results showed that the influence of bonding layer thickness on the surface wave dispersion is clear and could be applied to the NDE of bonding properties. In this paper, we employ an inversion algorithm to determine the thickness and the elastic properties of bonding layer in a bonded layered medium from the measured dispersion relation of the laser-generated surface waves. Laser ultrasonic experiments were performed on an epoxybonded copper-aluminum layered specimen with small bonding thickness. The received dispersive elastic wave signals were processed via the spectral analysis method to obtain the experimental dispersion curve. Inverse analyses of one parameter (thickness), two parameters (longitudinal and transverse wave velocities) and three parameters (density, longitudinal and transverse wave 
velocities) of the epoxy bonding layer were made. Discussions of the convergence and constraints of the inversion process are also included.

\section{Dispersion of laser-generated surface waves}

In laser ultrasonic measurements, a pulsed laser is usually employed to generate ultrasonic waves. The pulsed laser beam can be focused into a point or, in some cases, into a line segment or different geometry. A broadband optical detection system or a PZT type sensor is then adopted to receive the waves that are propagated through the medium. In the present work, an experimental study was performed on the propagation of generalized Rayleigh surface waves in an aluminum block with a copper surface layer. The dimensions of the aluminum block are $20 \times 20 \times 15 \mathrm{~cm}^{3}$, while the thickness of the copper layer is $1.02 \mathrm{~mm}$. The density of the aluminum specimen is $2698 \mathrm{~kg} \mathrm{~m}^{-3}$ and that of the copper is $8500 \mathrm{~kg} \mathrm{~m}^{-3}$. The copper layer was bonded carefully on the aluminum block with epoxy. The longitudinal and shear wave velocities of the copper and aluminum specimens were measured by the ultrasonic pulse-echo method and the results were

aluminum: $C_{\mathrm{L}}=6389.1 \mathrm{~m} \mathrm{~s}^{-1}, \quad C_{\mathrm{T}}=3097.8 \mathrm{~m} \mathrm{~s}^{-1}$,

copper: $C_{\mathrm{L}}=4744.2 \mathrm{~m} \mathrm{~s}^{-1}, \quad C_{\mathrm{T}}=2217.3 \mathrm{~m} \mathrm{~s}^{-1}$.

The density and the elastic wave velocities of the epoxy were also measured by the ultrasonic pulse-echo method. A small epoxy specimen was made from the same mixture as that used for bonding the copper thin plate and the aluminum block. The measured results of the epoxy sample were

$$
\begin{aligned}
& C_{\mathrm{L}}=2500.0 \mathrm{~m} \mathrm{~s}^{-1}, \quad C_{\mathrm{T}}=1112.2 \mathrm{~m} \mathrm{~s}^{-1}, \\
& \rho=1157.8 \mathrm{~kg} \mathrm{~m}^{-3} .
\end{aligned}
$$

The experimental set-up utilized in the present study is shown in Fig. 1. An Nd:YAG pulsed laser (QuantaRay, GCR-130) (wavelength $532 \mathrm{~nm}$ ) was utilized to generate elastic wave in the layered specimen. The duration of the laser pulse utilized was $10 \mathrm{~ns}$ and the energy carried was about $100 \mathrm{~mJ}$. The layered specimen was placed on a precision translation stage to accurately control the distance between the source and the receiver. An NBS conical transducer was utilized to measure the generated elastic wave signals from the laser sources. The received voltage signals from the conical transducer were then amplified by a preamplifier and recorded by a $100 \mathrm{MHz}$ digital oscilloscope (LeCroy 9314L). A trigger signal synchronized with the laser source was utilized to trigger the digital oscilloscope. The recorded signals were sent to a personal computer via GPIB.

For a point source acting on a layered half space, it is known that the wave signals received at two different

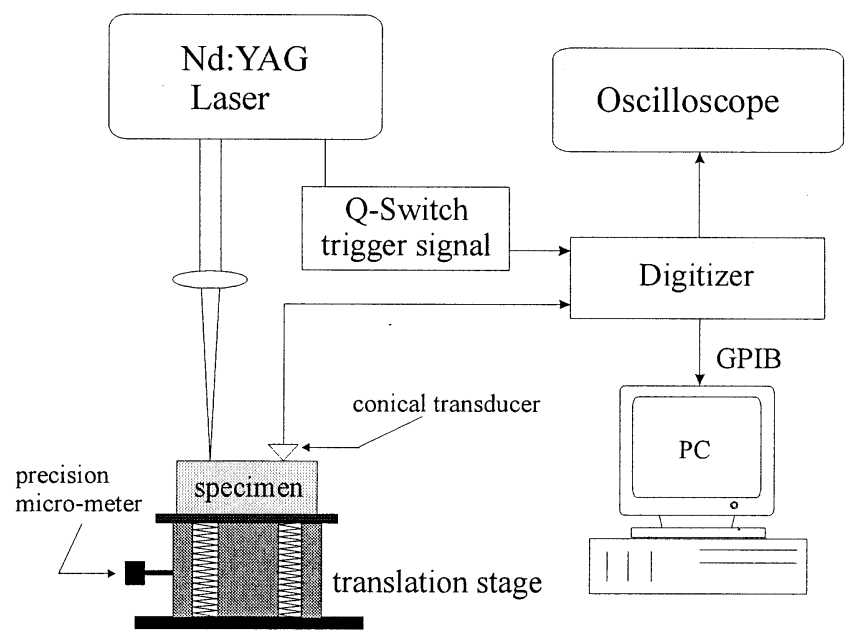

Fig. 1. Experimental set-up of the laser ultrasonic experiment.

receivers are different in both the amplitudes and phases due to the wave dispersion in the layered structure. Since the pulsed laser utilized in this study generates stable point sources, instead of using one point source and two receivers, one point receiver and two point sources were utilized. The advantage of the afore-mentioned alternative is that only one receiver is needed in the experiment. Further, the precision of the change of the source to receiver distance can be controlled by a precise translation stage.

Fig. 2 shows a typical wave signal (displacement perpendicular to the sample surface) received on the surface of the copper-aluminum specimen. The distance between the laser point source and receiver was $40.0 \mathrm{~mm}$. The vertical axis of Fig. 2 represents the relative amplitude of the displacement signal. Fig. 3 shows a similar

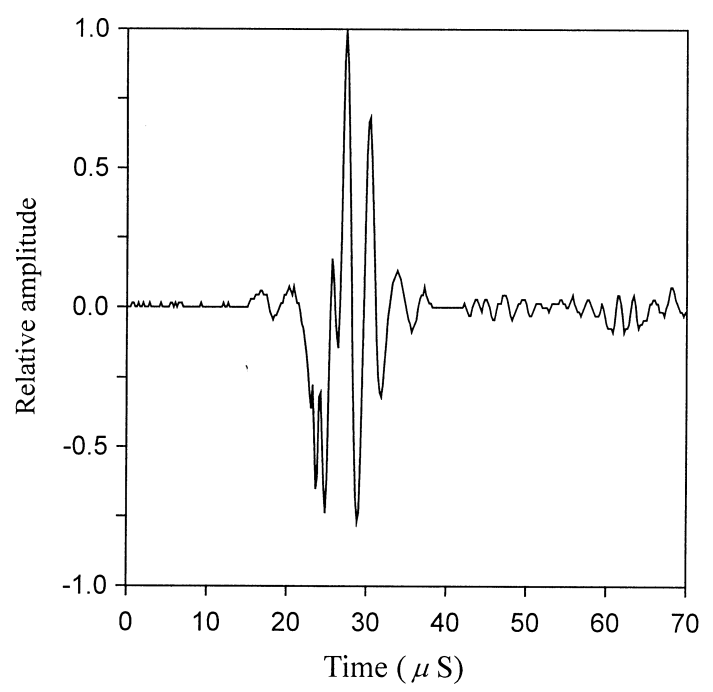

Fig. 2. The laser generated elastic wave signal received on the surface of an epoxy bonded copper-aluminum specimen. The distance between the laser point source and receiver is $40.0 \mathrm{~mm}$. 


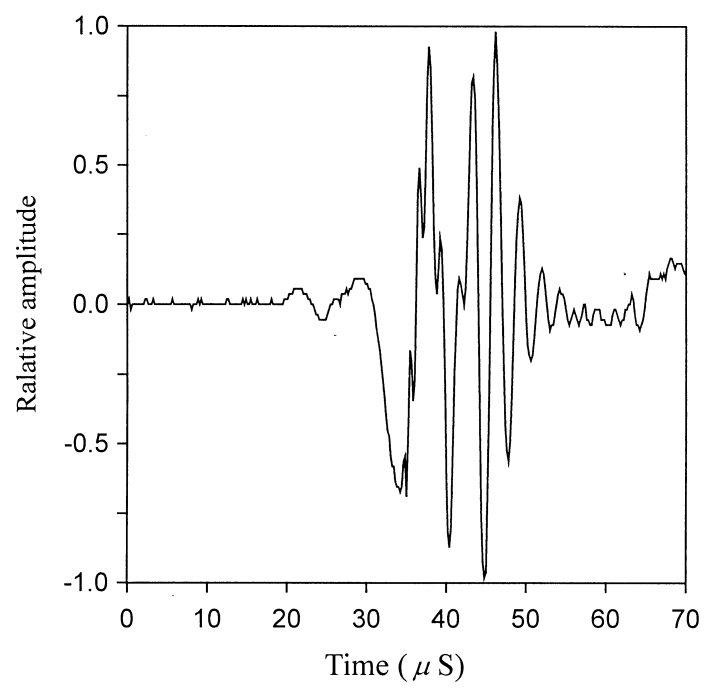

Fig. 3. The laser generated elastic wave signal received on the surface of an epoxy bonded copper-aluminum specimen. The distance between the laser point source and receiver is $76.3 \mathrm{~mm}$.

wave signal with source to receiver distance equal to $76.3 \mathrm{~mm}$.

\section{Spectral analysis of surface waves}

The phase difference $\phi$ of two wave signals received at two different positions $x_{1}$ and $x_{2}$ is equal to the phase angle of $S_{x_{1}, x_{2}}$ which is defined as [13]

$S_{x_{1}, x_{2}}=\frac{1}{n} \sum_{i=1}^{n}\left\{\left[R_{1}(f)\right]_{i}\left[R_{2}^{*}(f)\right]_{i}\right\}$.

In the above equation, $R_{1}(f), R_{2}(f)$ are the frequency spectrum of the wave signals received at two different positions $x_{1}$ and $x_{2}, f$ is the frequency of the harmonic wave, $R_{2}^{*}$ denotes the complex conjugate of $R_{2}$ and $n$ is the number of times the experiments repeated. On obtaining the phase difference $\phi$ as a function of frequency and distance $x_{1}-x_{2}$ from Eq. (1), the phase velocity $v$ can be obtained from the relation

$v=2 \pi f \frac{x_{1}-x_{2}}{\phi}$

On utilizing Eqs. (1) and (2), the received wave signals shown in Figs. 2 and 3 can be processed to obtain the phase velocity dispersion of the surface wave. The squares shown in Fig. 4 denote the measured phase velocity dispersion and the lines are the theoretical calculation of the phase velocity dispersion of the fundamental mode of surface waves in the layered specimen with different bonding thickness. The horizontal axis is the non-dimensional wave number $k_{x} H$ where $H$ is the thickness of the copper layer. The above-mentioned theoretical dispersion curves were calculated using a

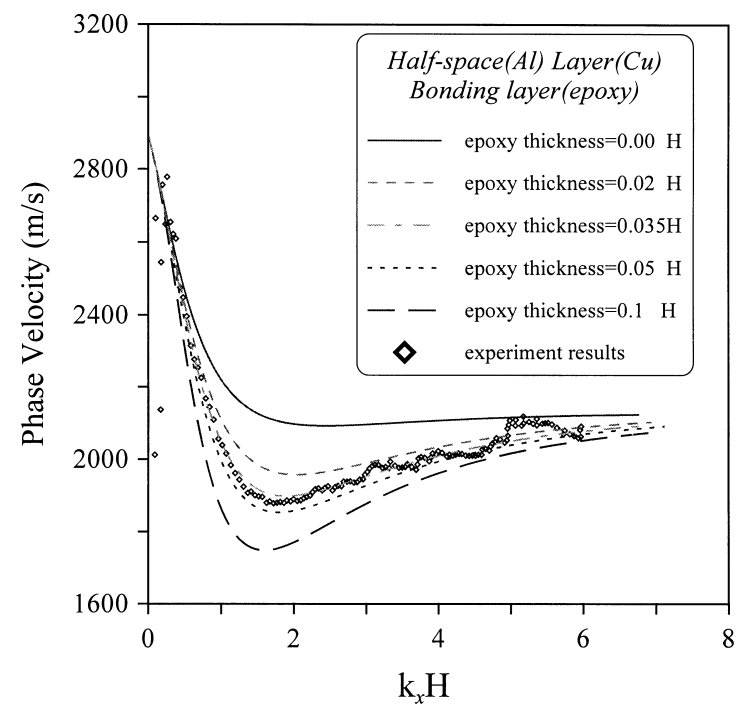

Fig. 4. The measured phase velocity dispersion (stars) and the calculated phase velocity dispersion (lines) of the fundamental mode of surface waves in the layered specimen with different bonding thickness.

general-purpose computer program [14] for the calculations of the dispersion curves of isotropic as well as anisotropic layered media. We note that, except for the scattered values of the measured phase velocities for $k_{x} H$ lower than about 0.3 , the general characteristics of the measured phase velocity dispersion agrees well with that of the calculated ones. The results shown in Fig. 4 indicate that the bonding thickness of the layered specimen lies between $0.02 \mathrm{H}$ and $0.05 \mathrm{H}$, which in this case is between $20 \mu \mathrm{m}$ and $50 \mu \mathrm{m}$.

\section{Inversion of bonding thickness and bonding properties}

The forward calculations of the phase velocity dispersions shown in the previous section showed that the shapes of the dispersion curves are strongly dependent on the thickness of the epoxy bonding layer, especially for the non-dimensional wave number between about 0.5 and 4 . We note that the thicker the epoxy bonding layer, the deeper the dip in the dispersion curve, i.e., the phase velocity of the fundamental surface wave mode decreases from the Rayleigh surface wave velocity of aluminum to a local minimum and then increases to that of copper (Fig. 4). The dip in the surface wave dispersion of a bonded layered half space provides a qualitative (and quantitative, if an inversion algorithm is implemented) indication of the thickness of the bonding layer. By inspection of the phase velocity dispersions in Fig. 4, one can easily find that the thickness of the bonding layer thickness is approximately equal to the value between $0.02 \mathrm{H}$ and $0.05 \mathrm{H}$. To further determine the thickness of the bonding layer quantitatively, an inverse algorithm is necessary. In addition, the disper- 
sion of the phase velocity of this problem is not only dependent on the thickness of the bonding layer but also on its elastic properties. Therefore, the elastic properties of the bonding layer can possibly be recovered by a suitable inverse algorithm.

\subsection{Inversion scheme}

An error function which defines the difference between the measured $\left(v_{\mathrm{m}}\right)$ and the guessed $\left(v_{\mathrm{g}}\right)$ phase velocities was defined as

$e=\frac{\sum_{i=1}^{N}\left[v_{\mathrm{m}}(i)-v_{\mathrm{g}}(i)\right]^{2}}{\sum_{i=1}^{N}\left[v_{\mathrm{m}}(i)\right]^{2}}$

where $i$ represents the discrete non-dimensional wave number and $N$ is the number of data points utilized in the inversion process.

In the inversion process, e.g. for recovering the bonding thickness, initial guesses of the bonding thickness were made first, then the forward computer program for calculating the phase velocity dispersion of surface wave in a layered medium was utilized to calculate the guessed phase velocities $\left(v_{\mathrm{g}}\right)$. The value of the error function can thus be obtained from Eq. (3). The true thickness of the bonding layer was determined using the simplex method [15] in this paper. A detailed flow chart of the simplex method can also be found in the paper by Chai and $\mathrm{Wu}[8]$.

\subsection{Inversion of bonding thickness}

50 measured phase velocities for $k_{x} H$ between 0.32 and 2.3 as those shown in Fig. 4 were adopted to determine the bonding thickness of the epoxy layer. Fig. 5 shows the dependence of the error function on the thickness of the epoxy bonding layer. The relationship shows that only one local minimum exists around the possible thickness of the bonding-layer and therefore, the inversion of the bonding thickness is believed to be very insensitive to the initial guesses of the thickness. Since only one parameter is to be inversely determined (the bonding-thickness), two initial guesses of the thickness are required in the simplex algorithm.

To test the influence of the initial guesses on the inverse result, a variety of initial guesses of the thickness ranging from $0.0 \mathrm{H}$ to $0.5 \mathrm{H}$ were utilized (Table 1). From Table 1, the true thickness can be obtained even for very poor initial guesses, for example, in the first pair of guesses, the true thickness does not lie between the initial guesses $0.0 \mathrm{H}$ to $0.01 \mathrm{H}$ and actually is far away from the initial guesses. The inversion result of the bonding-layer thickness was $0.0377 \mathrm{H}$. Fig. 6 shows the comparison of the measured and the calculated

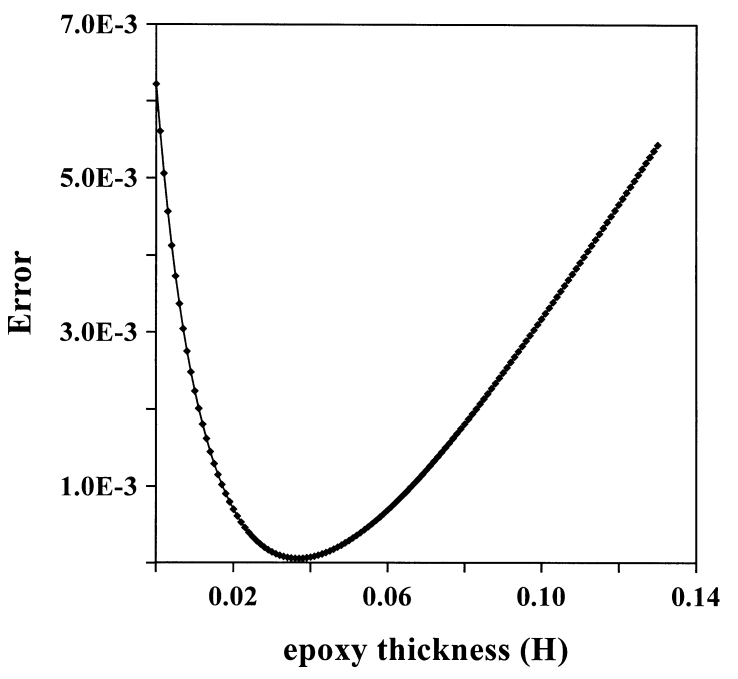

Fig. 5. The dependence of the error function on the thickness of the epoxy bonding layer.

Table 1

Lists of the initial guesses and the inverse results of the bonding thickness. Initial guesses of the thickness range from $0.0 \mathrm{H}$ to $0.5 \mathrm{H}$

\begin{tabular}{lllll}
\hline $\begin{array}{l}\mathrm{H} 1 \\
(H)\end{array}$ & $\begin{array}{l}\mathrm{H} 2 \\
(H)\end{array}$ & No. of calculation & $\begin{array}{l}\text { Thickness } \\
(H)\end{array}$ & $\begin{array}{l}\text { Error } \\
\left(\times 10^{-5}\right)\end{array}$ \\
\hline 0.00 & 0.01 & 24 & 0.03767 & 8.536 \\
0.00 & 0.02 & 22 & 0.03763 & 8.536 \\
0.01 & 0.03 & 22 & 0.03769 & 8.536 \\
0.03 & 0.05 & 17 & 0.03768 & 8.536 \\
0.05 & 0.07 & 18 & 0.03769 & 8.536 \\
0.07 & 0.09 & 19 & 0.03769 & 8.536 \\
0.12 & 0.20 & 21 & 0.03769 & 8.536 \\
0.20 & 0.30 & 19 & 0.03767 & 8.536 \\
0.30 & 0.50 & 18 & 0.03769 & 8.536 \\
0.00 & 0.50 & 24 & 0.03767 & 8.536 \\
\hline
\end{tabular}

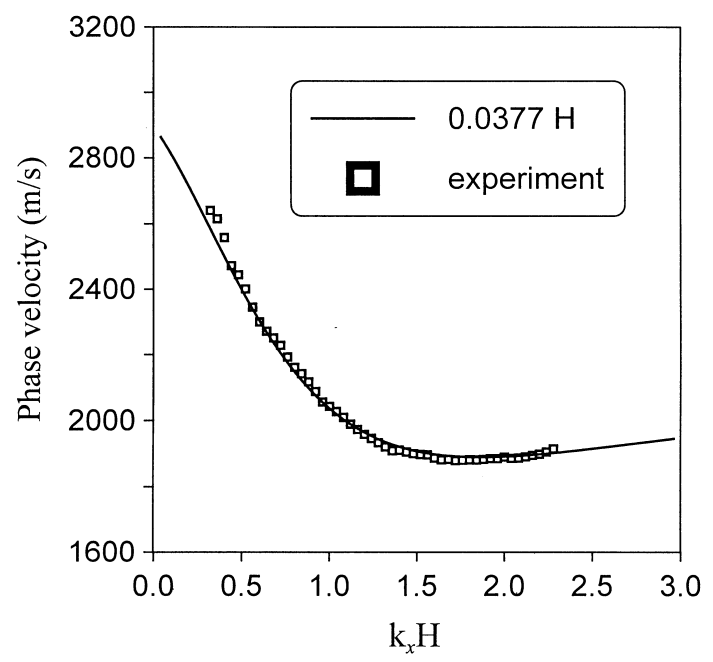

Fig. 6. The comparison of the measured and the calculated (with the bonding thickness equal to $0.0377 H$ ) phase velocity dispersions. 
(with the bonding thickness equal to $0.0377 \mathrm{H}$ ) phase velocity dispersions, the results show very good agreements between these two dispersion curves.

The measured phase velocities for $k_{x} H$ greater than 2.3 in Fig. 4 are slightly scattered around the theoretical curves. To check the influence of the scattered data on the inverse results, 100 measured phase velocities for $k_{x} H$ between 0.32 and 4.3 were adopted to determine the bonding thickness of the epoxy layer. In this case, the inversion result of the bonding-layer thickness determined is $0.0367 \mathrm{H}$, this is a little bit smaller than that obtained with 50 measured phase velocities. Since, the thickness of the copper layer was $1.02 \mathrm{~mm}$, the epoxy bonding layer was determined to be around $37 \mu \mathrm{m}$.

\subsection{Inversion of $C_{L}$ and $C_{T}$ of epoxy}

The longitudinal and transverse wave velocities are related to the elastic moduli of a solid, and therefore, measurements of the elastic wave velocities of a bonding layer are useful in accessing the bonding quality. In the following, we try to determine the longitudinal as well as the transverse wave velocities of the epoxy bonding layer inversely from the dispersive surface wave signals. In this case, we assume that the density and the thickness of the bonding layer are known.

Figs. 7 and 8 show the dependence of the error functions on the longitudinal and transverse wave velocities of the epoxy bonding layer, respectively. The result shown in Fig. 7 was obtained by fixing the transverse wave velocity at $C_{\mathrm{T}}=1112.2 \mathrm{~m} \mathrm{~s}^{-1}$, while that shown in Fig. 8 was obtained by fixing the longitudinal wave velocity at $C_{\mathrm{L}}=2500.0 \mathrm{~m} \mathrm{~s}^{-1} .50$ measured phase velocities for $k_{x} H$ between 0.32 and 2.3 in Fig. 4 were adopted in the inversion process. The relationships show that only one local minimum exists around the possible velocity variation of the bonding layer and therefore,

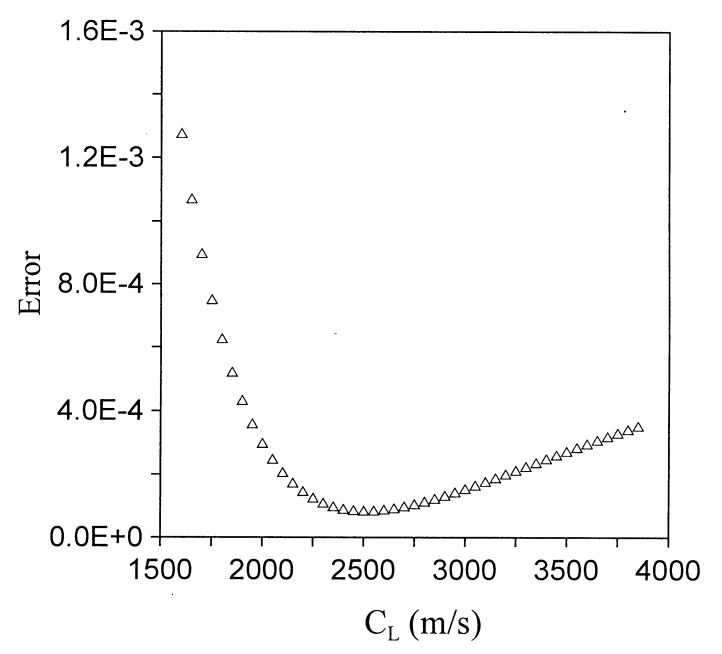

Fig. 7. The dependence of the error function on the longitudinal wave velocity with the transverse wave velocity fixed at $C_{\mathrm{T}}=1112.2 \mathrm{~m} \mathrm{~s}^{-1}$.

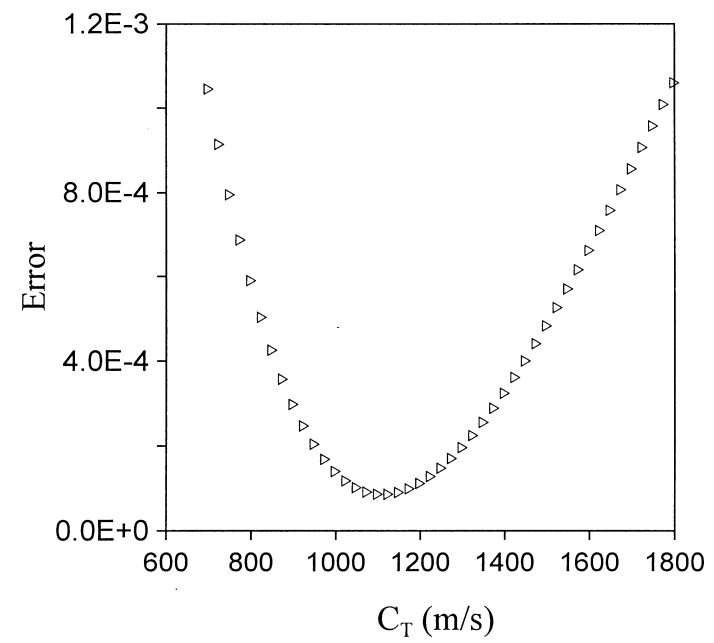

Fig. 8. The dependence of the error function on the transverse wave velocity with the longitudinal wave velocity fixed at $C_{\mathrm{L}}=$ $2500.0 \mathrm{~m} \mathrm{~s}^{-1}$.

the inversions of the wave velocities are believed to be relatively simple. In this case, three initial guesses of the velocities are required in the simplex algorithm. These initial guesses were constrained in the ranges $1600<C_{\mathrm{L}}<4100 \mathrm{~m} \mathrm{~s}^{-1}$ and $600<C_{\mathrm{T}}<2000 \mathrm{~m} \mathrm{~s}^{-1}$, which cover a wide range of the velocities of epoxy bonding materials. The inverse results of the longitudinal and transverse wave velocities of the epoxy bonding layer are shown in Table 2. Three sets of different initial guesses were made according to the constraints, and almost identical wave velocities of the epoxy layer were obtained. The determined longitudinal wave and transverse wave velocities of the epoxy layer were $C_{\mathrm{L}}=2567.0 \mathrm{~m} \mathrm{~s}^{-1}$ and $C_{\mathrm{T}}=1067.8 \mathrm{~m} \mathrm{~s}^{-1}$. The results show that there are small differences between the inversely determined and the ultrasonic measured elastic wave velocities. We note that the deviation error of the transverse wave velocity is greater than that of the longitudinal wave. However, the same is not true as the inversion parameters are increased. Fig. 9 shows the comparison of the measured and the calculated (based on the inversely determined wave velocities) phase velocity dispersions, the results are in good agreements.

\subsection{Inversion of $\rho, C_{L}$ and $C_{T}$ of epoxy}

To determine the elastic constants from the measured longitudinal and transverse wave velocities, the density of the material should be estimated first. Error of the density estimation may lead to considerable errors in the elastic constants. Therefore, in addition to the longitudinal and transverse wave velocities, it is practical and valuable if the density of the bonding layer can be recovered simultaneously from the inversion process.

To study the feasibility of simultaneous recovery of 
Table 2

Lists of the initial guesses and the inverse results of the longitudinal and transverse wave velocities of the bonding layer. The initial guesses are constrained in the ranges, $1600<C_{\mathrm{L}}<4100 \mathrm{~m} \mathrm{~s}^{-1}$ and $600<C_{\mathrm{T}}<2000 \mathrm{~m} \mathrm{~s}^{-1}$

\begin{tabular}{|c|c|c|c|c|c|c|c|}
\hline $\begin{array}{l}\text { Guessed } C_{\mathrm{L}} \\
\left(\mathrm{m} \mathrm{s}^{-1}\right)\end{array}$ & $\begin{array}{l}\text { Guessed } C_{\mathrm{T}} \\
\left(\mathrm{m} \mathrm{s}^{-1}\right)\end{array}$ & $\begin{array}{l}\text { Calculate } \\
\#\end{array}$ & $\begin{array}{l}C_{\mathrm{L}} \\
\left(\mathrm{m} \mathrm{s}^{-1}\right)\end{array}$ & $\begin{array}{l}C_{\mathrm{T}} \\
\left(\mathrm{m} \mathrm{s}^{-1}\right)\end{array}$ & $\begin{array}{l}\text { Error function value } \\
\left(\times 10^{-5}\right)\end{array}$ & $\begin{array}{l}C_{\mathrm{L}} \text { error } \\
(\%)\end{array}$ & $\begin{array}{l}C_{\mathrm{T}} \text { error } \\
(\%)\end{array}$ \\
\hline 1600 & 700 & & & & & & \\
\hline 2500 & 1100 & & & & & & \\
\hline 3200 & 1600 & 36 & 2566.77 & 1067.89 & 8.546 & 0.33 & 4.98 \\
\hline 1800 & 800 & & & & & & \\
\hline 2800 & 1200 & & & & & & \\
\hline 4100 & 2000 & 31 & 2566.93 & 1067.90 & 8.546 & 0.34 & 4.98 \\
\hline 1200 & 600 & & & & & & \\
\hline 1500 & 700 & & & & & & \\
\hline 1700 & 800 & 51 & 2567.31 & 1067.73 & 8.546 & 0.36 & 5.00 \\
\hline 2800 & 1245 & & & & & & \\
\hline 3200 & 1425 & & & & & & \\
\hline 3600 & 1600 & 46 & 2566.90 & 1067.84 & 8.546 & 0.34 & 4.99 \\
\hline
\end{tabular}

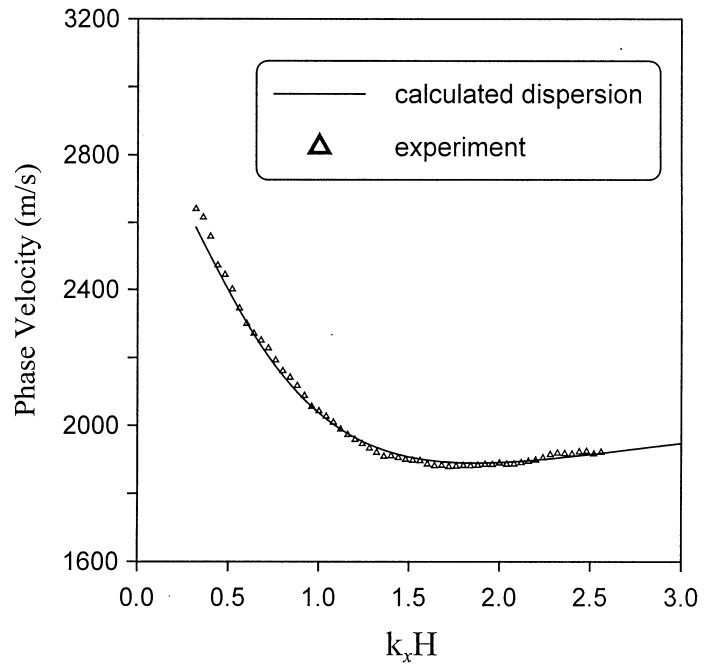

Fig. 9. The comparison of the measured and the calculated (based on the inversely determined wave velocities) phase velocity dispersions.

the density, longitudinal and transverse wave velocities, the first step is to investigate the dependence of the error functions on the various parameters.

Fig. 10 shows the dependence of the error function on the longitudinal and transverse wave velocities of the epoxy bonding layer when the density is fixed. The contour plot shows that although the descending of the error function value to the global minimum is not isotropic (with the same gradient from different descending direction), a global minimum does exist in this case. Fig. 11 is the dependence of the error function on the longitudinal wave velocity and the density, as the transverse velocity is fixed. This contour plot also shows the existence of a global minimum. A similar result was also found for the case of error function vs. the density and the transverse wave velocity.

50 measured phase velocities for $k_{x} H$ between 0.32 and 2.3 in Fig. 4 were then adopted in the inversion process. In this case, four sets of initial guesses are

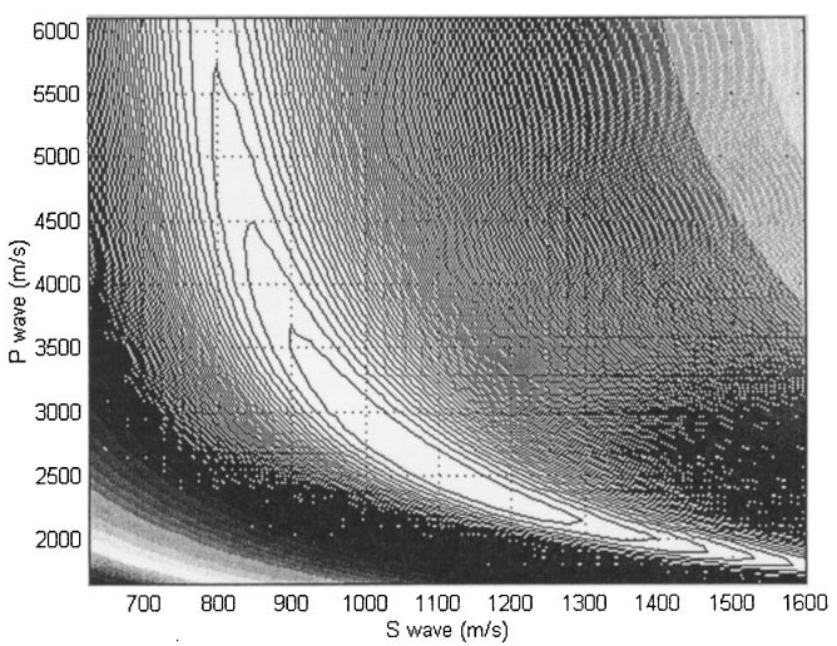

Fig. 10. The dependence of the error function on the longitudinal and transverse wave velocities of the epoxy bonding layer when the density is fixed.

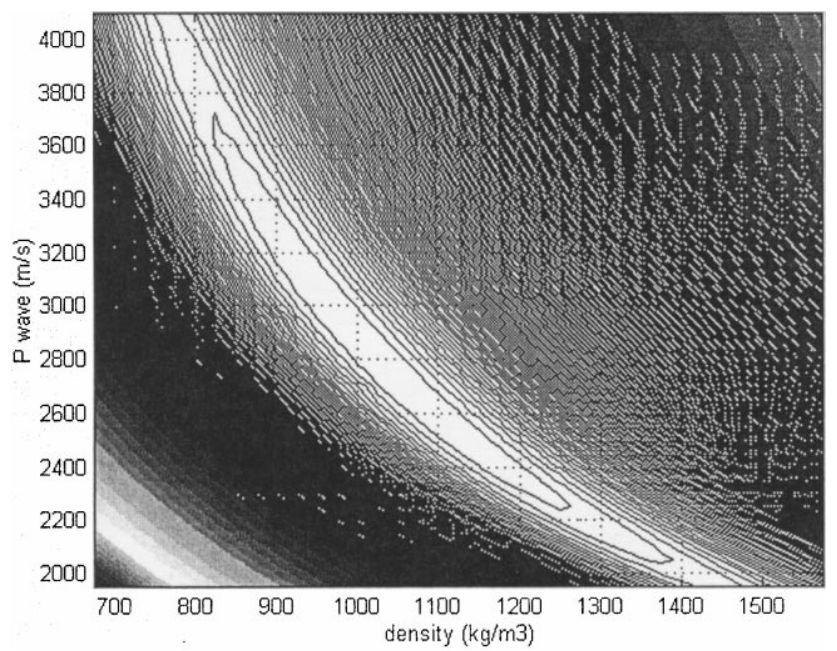

Fig. 11. The dependence of the error function on the longitudinal wave velocity and density of the epoxy bonding layer when the transverse wave velocities is fixed. 
required in the simplex algorithm. The inverse results of density, longitudinal and transverse wave velocities of the epoxy bonding layer are shown in Table 3. The results show that although the values of the error function are very small in these three sets of initial guesses, the inversion results of the density, the longitudinal wave velocity and the transverse wave velocity have about $1-6 \%$ errors. To achieve more accurate inversion results, much smaller error tolerance should be given, however, this increases the inversion time dramatically. To accelerate and improve the inversion accuracy, reasonable constraints on the inversion parameters were found to be practical and efficient. The results of Table 4 were obtained with the following constraints made in the inversion process, $2300 \mathrm{~m} \mathrm{~s}^{-1}<C_{\mathrm{L}}<2800$ $\mathrm{m} \mathrm{s}^{-1}, \quad 900 \mathrm{~m} \mathrm{~s}^{-1}<C_{\mathrm{T}}<1300 \mathrm{~m} \mathrm{~s}^{-1}, \quad 1000 \mathrm{~kg} \mathrm{~m}^{-3}<\rho$ $<1250 \mathrm{~kg} \mathrm{~m}^{-3}$. We note that the constraints cover most of the elastic wave velocities and density of the commercial available epoxy. On comparing the results of
Tables 3 and 4, it is seen that the accuracy of the inversion results improves significantly when the constraints are made, especially for the density inversion (Table 4).

\section{Discussion}

With the successful experiences in the inversion of the thickness and the elastic properties of the bonding epoxy from the laser-generated wave signals, one may expect to determine the afore-mentioned unknowns simultaneously. However, preliminary studies showed that the current approach could not obtain a unique and correct inversion. The inversion process may lead to a wrong solution but give a theoretical dispersion curve, which is very close to the measured dispersion curve. For example, combination of a large thickness and low wave velocities results to a similar dispersion curve (the funda-

Table 3

Lists of the initial guesses and the inverse results of the density, the longitudinal and transverse wave velocities of the bonding layer (with no constraints)

\begin{tabular}{|c|c|c|c|c|c|c|c|c|c|}
\hline $\begin{array}{l}\text { Guess } d \\
\left(\mathrm{~kg} \mathrm{~m}^{-3}\right)\end{array}$ & $\begin{array}{l}\text { Guess } C_{\mathrm{L}} \\
\left(\mathrm{m} \mathrm{s}^{-1}\right)\end{array}$ & $\begin{array}{l}\text { Guess } C_{\mathrm{T}} \\
\left(\mathrm{m} \mathrm{s}^{-1}\right)\end{array}$ & $\begin{array}{l}\text { Error function } \\
\left(\times 10^{-5}\right)\end{array}$ & $\begin{array}{l}\text { Density } \\
\left(\mathrm{kg} \mathrm{m}^{-3}\right)\end{array}$ & $\begin{array}{l}C_{\mathrm{L}} \\
\left(\mathrm{m} \mathrm{s}^{-1}\right)\end{array}$ & $\begin{array}{l}C_{\mathrm{T}} \\
\left(\mathrm{m} \mathrm{s}^{-1}\right)\end{array}$ & $\begin{array}{l}\text { Density error } \\
(\%)\end{array}$ & $\begin{array}{l}C_{\mathrm{L}} \text { error } \\
(\%)\end{array}$ & $\begin{array}{l}C_{\mathrm{T}} \text { error } \\
(\%)\end{array}$ \\
\hline \multicolumn{10}{|c|}{ No constraint } \\
\hline 1100 & 2100 & 1000 & & & & & & & \\
\hline 1300 & 3200 & 1400 & & & & & & & \\
\hline 1500 & 2700 & 1100 & & & & & & & \\
\hline 1700 & 2400 & 800 & 8.568 & 1231.48 & 2430.25 & 1078.47 & 6.36 & 2.79 & 3.03 \\
\hline 1000 & 1600 & 800 & & & & & & & \\
\hline 1200 & 2400 & 1200 & & & & & & & \\
\hline 1400 & 2800 & 1100 & & & & & & & \\
\hline 1100 & 2500 & 900 & 8.540 & 1115.72 & 2580.54 & 1132.14 & 3.63 & 3.22 & 1.79 \\
\hline 1000 & 1600 & 900 & & & & & & & \\
\hline 1300 & 2400 & 1200 & & & & & & & \\
\hline 1400 & 2800 & 1300 & & & & & & & \\
\hline 1100 & 2200 & 1000 & 8.518 & 1170.05 & 2608.76 & 1070.05 & 1.05 & 4.35 & 3.78 \\
\hline
\end{tabular}

Table 4

Lists of the initial guesses and the inverse results of the density, the longitudinal and transverse wave velocities of the bonding layer (with constraints)

\begin{tabular}{|c|c|c|c|c|c|c|c|c|c|}
\hline $\begin{array}{l}\text { Guess } d \\
\left(\mathrm{~kg} \mathrm{~m}^{-3}\right)\end{array}$ & $\begin{array}{l}\text { Guess } C_{\mathrm{L}} \\
\left(\mathrm{m} \mathrm{s}^{-1}\right)\end{array}$ & $\begin{array}{l}\text { Guess } C_{\mathrm{T}} \\
\left(\mathrm{m} \mathrm{s}^{-1}\right)\end{array}$ & $\begin{array}{l}\text { Error function } \\
\left(\times 10^{-5}\right)\end{array}$ & $\begin{array}{l}\text { Density } \\
\left(\mathrm{kg} \mathrm{m}^{-3}\right)\end{array}$ & $\begin{array}{l}C_{\mathrm{L}} \\
\left(\mathrm{m} \mathrm{s}^{-1}\right)\end{array}$ & $\begin{array}{l}C_{\mathrm{T}} \\
\left(\mathrm{m} \mathrm{s}^{-1}\right)\end{array}$ & $\begin{array}{l}\text { Density error } \\
(\%)\end{array}$ & $\begin{array}{l}C_{\mathrm{L}} \text { error } \\
(\%)\end{array}$ & $\begin{array}{l}C_{\mathrm{T}} \text { error } \\
(\%)\end{array}$ \\
\hline Constraints & \multicolumn{9}{|c|}{$d=1000 \sim 1250 \quad C_{\mathrm{p}}=2300 \sim 2800 \quad C_{\mathrm{s}}=900 \sim 1300$} \\
\hline 1100 & 2400 & 1000 & & & & & & & \\
\hline 1150 & 2700 & 1250 & & & & & & & \\
\hline 1200 & 2600 & 1100 & & & & & & & \\
\hline 1050 & 2450 & 950 & 8.514 & 1153.46 & 2566.72 & 1096.28 & 0.37 & 2.66 & 1.43 \\
\hline 1000 & 2400 & 1000 & & & & & & & \\
\hline 1150 & 2600 & 1100 & & & & & & & \\
\hline 1200 & 2700 & 1150 & & & & & & & \\
\hline 1100 & 2500 & 1050 & 8.508 & 1147.21 & 2594.42 & 1096.48 & 0.91 & 3.77 & 1.41 \\
\hline 1100 & 2700 & 1050 & & & & & & & \\
\hline 1150 & 2550 & 1200 & & & & & & & \\
\hline 1200 & 2400 & 1250 & & & & & & & \\
\hline 1050 & 2350 & 1000 & 8.556 & 1125.24 & 2565.27 & 1119.42 & 2.81 & 2.61 & 0.64 \\
\hline
\end{tabular}




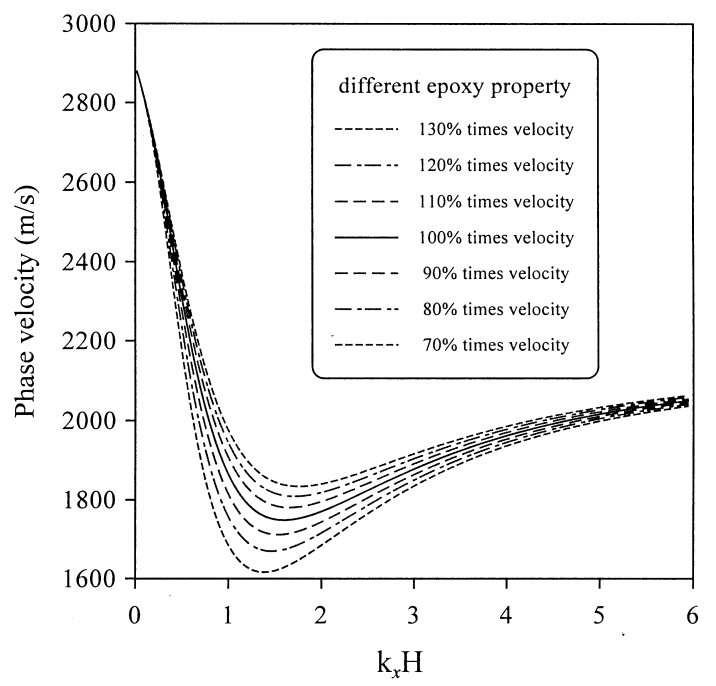

Fig. 12. Influence of the elastic properties of the bonding layer on the dispersion curve of the surface wave.

mental mode of the generalized Rayleigh wave) with that of a small thickness and high wave velocities. The uniqueness problem of the inversion may arise from the assumption of the current approach, i.e., only the fundamental mode of the generalized surface wave was utilized in the matching between the theoretical and the measured dispersion curves. To solve the uniqueness problem in the inversion process, an experimental method for measuring both the fundamental and the first higher modes of the generalized surface wave is awaited.

To understand the influence of the elastic properties of the bonding layer on the dispersion curve of the surface wave, numerical simulations were also conducted. Fig. 12 shows the numerical result for the case of different bonding layer properties. The longitudinal and transverse wave velocities of the bonding layer were varied from $70 \%$ to $130 \%$ of the true velocities of the epoxy layer. The results show that the characteristics of the dispersion curves are similar. Therefore, it is believed that the current inversion scheme can be applied to a wide range of different bonding materials.

\section{Concluding remarks}

Laser ultrasonic experiments were performed and the acquired surface wave signals were processed in the frequency domain to obtain the dispersion relation of the phase velocity of an epoxy bonded layered specimen. A computer program for the calculation of the phase velocity dispersion of general isotropic and/or anisotropic layered media was utilized to explore the influence of the epoxy bonded layer. The dependence of the error function on the inversion parameters, such as the thickness, elastic wave velocities and the density of the bonding layer were calculated first. Inversions of the bonding layer thickness and the elastic wave velocities of the epoxy layer were then performed. The results showed that for the case of thickness or elastic property inversions, only one global minimum exists in each of the inversion problems. The inversion results demonstrated that the thickness or the elastic properties of the bonding layer could be successfully determined.

\section{Acknowledgement}

The authors thank the financial support of this research from the National Science Council of ROC through the grant NSC86-2212-E-002-078.

\section{References}

[1] C.B. Scruby, L.E. Drain, Laser Ultrasonics: Techniques and Applications, IOP Publishing Ltd, Bristol, 1990.

[2] D.A. Hutchins, K. Lundgren, S.B. Palmer, A laser study of the transient Lamb waves in thin materials, J. Acoust. Soc. Am. 85 (4) (1989) 1441.

[3] R.J. Dewhurst, C. Edwards, A.D.W. Mckie, S.B. Palmer, Estimation of the thickness of thin metal sheet using laser generated ultrasound, Appl. Phys. Lett. 51 (1987) 1066.

[4] H. Nakano, S. Nagai, Laser generation of anti-symmetric Lamb waves in thin plates, Ultrasonics 29 (1991) 230.

[5] M. Veidt, W. Sachse, Ultrasonic point-source/point-receiver measurements in thin specimens, J. Acoust. Soc. Am. 96 (4) (1994) 2318.

[6] K. Castagnede, K.Y. Kim, W. Sachse, M.O. Thompson, Determination of the elastic constants of anisotropic materials using lasergenerated ultrasonic signals, J. Appl. Phys. 70 (1) (1991) 150.

[7] T.-T. Wu, J.-F. Chai, Propagation of surface waves in anisotropic solids: theoretical calculation and experiment, Ultrasonics 32 (1) (1994) 21.

[8] J.-F. Chai, T.-T. Wu, Determinations of anisotropic elastic constants using laser generated surface waves, J. Acoust. Soc. Am. 95 (6) (1994) 3232.

[9] P.A. Doyle, C.M. Scala, Ultrasonic measurement of elastic constants for composite overlays, Rev. Prog. in QNDE 10B (1991) 1453.

[10] M.A. Johnson, Y.H. Berthelot, P.H. Brodeur, L.A. Jacobs, Investigation of laser generation of Lamb waves in copy paper, Ultrasonics 34 (1996) 703.

[11] L.P. Scudder, D.A. Hutchin, N. Guo, Laser-generated ultrasonic guided waves in fiber-reinforced plates - theory and experiment, IEEE Trans. Ultra. Ferro. Freq. Control 43 (5) (1996) 870.

[12] T.-T. Wu, Y.-C. Chen, Dispersion of laser generated surface waves in an epoxy-bonded layered medium, Ultrasonics 34 (1996) 793.

[13] S. Nazarian, M.R. Desai, Automated surface wave method: field testing, Journal of Geotechnical Engineering 119 (7) (1992) 1094.

[14] Y.-C. Chen, Dispersion of surface waves in an anisotropic layered medium, M.S. Thesis, National Taiwan University, 1994.

[15] J.A. Nelder, R. Mead, A simplex method for function minimization, Computer Journal 7 (1965) 308. 